\title{
Los procesos de formación del docente de inglés en el contexto global
}

\author{
ASTRID RAMÍREZ VALENCIA** \\ MAGNOLIA RAMÍREZ VALENCIA** \\ LUCÍA BUSTAMANTE VÉLEZ****
}

Recepción: 28 de agosto de 2018

Aprobación: 30 de noviembre de 2018

Forma de citar este artículo: Ramírez, A. Ramírez, M. \& Bustamante, L. (2019). Los procesos de formación del docente de inglés en el contexto global. Cuadernos de Lingüística Hispánica, (33), 153-168.

đoil 10.19053/0121053X.n33.2019.7217

* El artículo constituye una reflexión a partir de los procesos pedagógicos e investigativos sobre la práctica del docente de inglés.

** Magíster en Enseñanza del Inglés como Lengua Extranjera, Universidad Distrital; doctoranda en Lenguaje y Cultura, Universidad Pedagógica y Tecnológica de Colombia. Docente-investigadora de la Licenciatura en Educación Básica con Énfasis en Inglés, Universidad Distrital. Correo electrónico: astramirez@gmail.com. (D) https://orcid.org/0000-0002-3025-5982

*** Magíster en Comunicación, Educación y Literatura, Universidad Distrital. Correo electrónico: magnolar@yahoo.com (Di) https://orcid.org/0000-0003-0190-1786

****Doctora en Lenguaje y Cultura, Universidad Pedagógica y Tecnológica de Colombia; integrante del grupo de investigación Corporación "Si Mañana Despierto" para la Creación e Investigación de la Literatura y las Artes. Docente Escuela de Idiomas, UPTC. Editora Revista Cuadernos de Lingüística Hispánica. Coordinadora del Doctorado en Lenguaje y Cultura y de la Maestría en Lingüística. Correo electrónico: lucia.bustamante@uptc.edu.co (Di) https://orcid.org/0000-0002-7227-2019 


\section{Resumen}

El artículo presenta una discusión acerca del proceso de formación que se espera debe recibir el futuro profesor de inglés, a la luz del fenómeno de la globalización, con las exigencias y los retos impuestos por esta nueva condición. Expone un bosquejo acerca de las implicaciones pedagógicas de este fenómeno y los referentes teóricos que soportan la relación entre la globalización, la caracterización de este nuevo docente y las exigencias que le impone esta condición a su proceso de formación. El proceso metodológico se sustenta en la reflexión teórica de estas consideraciones. Como resultado plantea algunos lineamientos para ser tenidos en cuenta en el proceso de formación de un docente de inglés en el contexto global.

Palabras clave: proceso de formación, docente, globalización.

\section{The Processes of English Teacher Training in the Global Context}

\section{Abstract}

The article presents a discussion about the training process expected to be received by the future English teacher, from the perspective of the globalization, showing the demands and challenges imposed by this new condition. It shows an outline about the pedagogical implications of this event and the theoretical references that support the relationship between globalization, the characterization of this new teacher and the demands imposed by this condition, to his/her training process. The methodological process is based on the theoretical reflection of these considerations. As a result, it raises some guidelines to be taken into account in the process of training an English teacher in the global context.

Keywords: training process, teacher, globalization. 


\section{Les processus de formation de l'enseignant d'anglais dans un contexte mondial}

\section{Résumé}

Larticle présente une discussion par rapport au processus de formation que l'enseignant d'anglais est censé de recevoir, à la lumière du phénomène de la mondialisation, avec les exigences et les défis imposés par cette nouvelle condition. L'article expose également une exquise par rapport aux implications pédagogiques de ce phénomène et les référents théoriques qui supportent le rapport entre la mondialisation, la caractérisation de ce nouvel enseignant et les exigences qui lui impose cette nouvelle condition à son processus de formation. Le processus méthodologique est fondé sur la réflexion théorique de ces considérations. Comme résultat, on aborde quelques lignes directrices à considérer dans le processus de formation d'un enseignant dans le contexte mondial.

Mots clés : processus du formation, enseignant, mondialisation.

\section{Os processos de formação do professor de inglês no contexto global}

\section{Resumo}

0 artigo apresenta uma discussão sobre 0 processo de formação que o futuro professor de inglês deverá receber, à luz do fenômeno da globalização, com as demandas e desafios impostos por essa nova condição. Expõe um esboço sobre as implicações pedagógicas desse fenômeno e os referentes teóricos que sustentam a relação entre a globalização, a caracterização desse novo professor e as demandas que essa condição impõe ao seu processo de formação. 0 processo metodológico baseia-se na reflexão teórica dessas considerações. Como resultado, propõe algumas diretrizes a serem levadas em conta no processo de formação de um professor de inglês no contexto global.

Palavras-chave: processo, formação, professor, globalização. 


\section{Introducción}

En la actualidad se habla mucho sobre la globalización y su incidencia en la educación, pero poco se discute acerca de la formación que debe recibir el futuro docente de inglés en esta nueva condición. En los párrafos siguientes se abordará esta cuestión, explicando diferentes posturas enmarcadas por la influencia de las nuevas condiciones impuestas por este fenómeno.

Existen diferentes aspectos inherentes a la globalización, los cuales modifican el proceso educativo impartido en las instituciones formadoras de los docentes de inglés y a partir de los cuales surgen interrogantes como los planteados por Kumaravadivelu (2006), quien indaga acerca del nuevo contexto global enfrentado por la educación del profesor de inglés en estos días. Este autor sugiere el acercamiento a la teorización, investigación y reflexión con autonomía, pragmatismo y evaluación crítica, por parte de quienes se están formando y también de los futuros docentes de inglés, lo cual se espera que sea alcanzado durante su periodo de formación.

Esta reflexión se relaciona de forma directa con el ejercicio docente del futuro profesor de inglés, con especial énfasis en la discusión sobre su desempeño en la práctica en la escuela. Se sugiere que este ejercicio se lleve a cabo de manera grupal, es decir, mediante un análisis colectivo de las situaciones que se presentan en el contexto escolar, para encontrar una solución a las discusiones planteadas en la comunidad educativa.

Al respecto, Zabalza (2004) afirma que la revisión sistemática del proceso de formación profesional se puede realizar a través de sistemas de observación y evaluación del mismo, que vinculen la teoría y la práctica profesional. Con base en esta sugerencia, se exponen las reflexiones surgidas frente a la práctica docente, que puedan conducir a la puesta en marcha de propuestas de investigación, las cuales permitan resolver las problemáticas detectadas en los espacios escolares.

Esto se logra, siempre y cuando el profesor de inglés en proceso de formación considere muy importante la práctica, por ser el espacio que le brinda la oportunidad de conocer la realidad del aula y, al mismo tiempo, le permite crecer profesionalmente, al contrastar las teorías aprendidas con su práctica pedagógica.

\section{Fundamentación teórica}

Hay que resaltar la importancia de ser consciente acerca de la necesidad que tiene el futuro profesor de inglés de adueñarse de su proceso de formación, lo cual le permitirá ir más allá de las fuentes brindadas por las instituciones formadoras. Kumaravadivelu 
(2003) plantea que la autonomía representa la libertad para actuar, el deseo y la habilidad de pensar de manera independiente y de actuar con responsabilidad. En otras palabras, tanto el estudiante como el docente requieren concientizarse del valor de ser dueños de sus acciones pedagógicas.

El futuro docente de inglés está llamado a ser constructor de su propio proceso de formación, el cual se verá fortalecido en la medida que exista un compromiso permanente de ser copartícipe de este proceso, que resultará en el mejoramiento de su desempeño pedagógico.

Hallak (2008), por su parte, afirma que el profesor en proceso de formación debe ser creativo cuando enfrenta distintas situaciones en la escuela, para que pueda dar respuestas coherentes y acordes con los principios teóricos adquiridos durante su proceso de formación. Es decir, dichos principios teóricos deben orientarlo sobre cómo actuar, aunque permitiéndole producir sus propias teorías, resultado de las reflexiones sobre las situaciones presentadas en conjunto con su profesor director de práctica.

En concordancia con esta perspectiva, León (2004) manifiesta que el docente está llamado a ser un productor de conocimiento y un profesional capaz de convertir la voluminosa información en conocimiento útil, lo cual se traduce en la generación de ideas y teorías con las cuales emprenda acciones que solucionen las problemáticas del aula.

Adicionalmente, se espera que el profesor de inglés sea formado con un carácter flexible frente a los cambios producidos a partir de fenómenos como la globalización. A propósito, Sánchez (s.f.) y Peña (2003) afirman que el docente debe ser realista, coherente, flexible y viable, y que la academia debe promocionar una enseñanza activa, reflejo de las concepciones y experiencias de los profesores en proceso de formación y de sus formadores, que propicien estrategias cognitivas y metacognitivas para el aprendizaje de los docentes; de tal manera que su tutor desarrolle procesos de asesoramiento y apoyo facilitadores del aprendizaje entre iguales.

Esto significa que el profesor director de práctica también debe ser un provocador del conocimiento, un generador de situaciones donde los estudiantes estén involucrados, y alguien comprometido con el aprendizaje de un saber, que permita, de igual manera, aprender de su actividad pedagógica de forma amplia, involucrando incluso a sus estudiantes en la búsqueda de este propósito (Jiménez, 2014). 
Por esta razón, el futuro profesor de inglés debe ser consciente de que su proceso de aprendizaje nunca termina y que, por el contrario, la experiencia adquirida es fuente de conocimiento e indagación constante, de tal manera que esta experticia lo lleve a elaborar teorías surgidas a partir del contacto con la realidad. En este sentido, debe encontrar canales de divulgación de sus hallazgos, dados por los avances tecnológicos, promovidos de manera amplia, a partir del fenómeno de la globalización.

Esto se logra, si el docente de inglés está comprometido con los procesos de reflexión sobre su desempeño pedagógico. Biggs (2006) observa al respecto, que promover la reflexión del maestro sobre su propia práctica permite no solo visibilizarla, sino potenciarla. Los profesores tienen siempre algún tipo de teoría de la enseñanza, pero suele estar implícita, por eso al final queda sin examinar. Por esta razón, los futuros maestros deben incorporar la reflexión crítica en su actividad diaria, de manera que analicen, consideren y razonen sobre los acontecimientos de su entorno, para alcanzar así un desempeño profesional mucho más efectivo y exitoso (García y García, 2014).

A propósito, Brunner (2001) señala que se pueden reconocer algunas situaciones ilustradoras de los nuevos escenarios educativos dados por la globalización, que generan otros modos de formación para el docente de inglés.

\subsection{Condiciones planteadas por la globalización}

Los procesos mencionados por Brunner (2001) están caracterizados por varias condiciones: la primera, acomodarse a las transformaciones del conocimiento. Aquí es necesario aclarar que el conocimiento ha dejado de ser lento, escaso y estable, y, frente a la existencia de múltiples formas de acceso a la información, la escuela se ve abocada a abandonar los tradicionales métodos de enseñanza. Consecuentemente, el currículo ofrecido al futuro profesor de inglés se debe planear para que esté en capacidad de adaptarse a los cambios que la globalización ha provocado en el conocimiento y en la enseñanza de la lengua extranjera.

Debido a estos cambios, la escuela ya no es considerada como único escenario de aprendizaje, porque esta era nos provee de varios contextos educativos y ambientes generadores de aprendizaje, los cuales ofrecen condiciones más cómodas para el aprendiz, en los aspectos social, académico y económico. Por otro lado, como el docente y los libros de ejercicios ya no son las únicas fuentes de conocimiento y comunicación, la escuela debe adaptarse a este contexto, ofreciendo espacios de aprendizaje más flexibles, con el objetivo de facilitar el acceso y la recepción del conocimiento. Todo esto exige al profesor de inglés una formación que se guíe por los requerimientos del contexto global. 
Según Brunner (2001), hay, por lo menos, cuatro perspectivas interconectadas que pueden ayudar a entender la rápida evolución del contexto global:

El conocimiento se está transformando, no es estático y frecuentemente se redefine; hay una resignificación de los espacios educativos existentes, el profesor de inglés debe ser consciente de las nuevas condiciones de la enseñanza dadas por la globalización y la escuela debe prepararse para satisfacer las nuevas condiciones donde actuará (Brunner, 2001, p. 2).

La primera condición, la transformación del conocimiento, lleva al profesor de inglés a tener acceso al conocimiento creándole también nuevos saberes de manera insospechada y completamente diferente a la tradicional. En este sentido, el futuro profesor de inglés será consciente de que ningún conocimiento es suficiente frente a los avances de la ciencia, la pedagogía y, ante todo, de la tecnología; en consecuencia, debe plantear acciones que le permitan estar actualizado constantemente.

La segunda condición, la resignificación de los espacios, apunta a utilizar otros ambientes de aprendizaje, que ofrezcan nuevas oportunidades de enseñanza y creen la necesidad de tener en cuenta otros criterios en la situación de enseñanza ofrecida por el maestro. Busca, así, involucrarse con otros contextos, a pesar de las tensiones que genera la ruptura con los tradicionales espacios de enseñanza. Cabero (2002) concuerda con esta perspectiva al aclarar que, en la actualidad, se están creando entornos de enseñanza facilitadores para los usuarios (profesores y estudiantes) realizadores de actividades formativas, independientemente del espacio y el tiempo donde se encuentren. Estos nuevos entornos ofrecen al estudiante la posibilidad de una elección real respecto a cuándo, cómo y dónde estudiar, condición favorecedora del proceso de aprendizaje individual, a su propio ritmo y en sus propias circunstancias.

La tercera condición, la consciencia docente frente a la globalización, implica que el futuro profesor de inglés debe estar preparado para el reto impuesto por las nuevas situaciones que esta era genera, pues presenta unas características particulares donde el profesor es un facilitador del aprendizaje, cuyo conocimiento nunca termina por la velocidad con que cambia la información, lo que implica que debe estar constantemente actualizado e involucrado en el saber.

A tal efecto, se debe formar al futuro profesor de inglés para que esté dispuesto a afrontar los cambios, pues día a día, las transformaciones en los conceptos se darán con menor tiempo. Al respecto, Fernández (2003) señala la necesidad de ofrecer en la Universidad un nuevo modelo de formación, lo cual implica un cambio estructural en la 
educación superior. Lo anterior se traduce en que es fundamental ofrecer programas de formación de docentes que respondan adecuadamente a las necesidades de la sociedad actual y a los cambios sociales y económicos que exige, para llevar al futuro docente de inglés a crear un proceso de formación sostenible; es decir, pertinente para el momento en que ejerza su labor.

Finalmente, Brunner (2001) plantea una cuarta condición: la preparación de la escuela para satisfacer las necesidades de la globalización. De allí surge la necesidad de replantear al docente y analizar de manera más detallada su campo de acción, el cual gira alrededor de todos los hechos ocurridos dentro o fuera de la escuela. Por esta razón, su configuración estará fundamentada en la investigación, la reflexión y la transcripción de los acontecimientos transcurridos en su actividad diaria.

Se evidencia la conveniencia de dejar de formar docentes que sean solamente trasmisores de conocimiento, para crear profesores que estén pendientes de las situaciones presentes dentro y fuera de la escuela, que inquieten a sus estudiantes acerca de las mismas; profesores conscientes de su importancia individual, colectiva y ciudadana, que formen a sus alumnos como verdaderos ciudadanos, para que el mundo pueda convertirse en un sitio más amigable, con personas responsables y cuidadosas con el medio ambiente, que dejen un mejor legado a sus descendientes.

También es pertinente tener en cuenta los planteamientos establecidos por Hallak (2008), miembro notable de la Organización de las Naciones Unidas para la Educación, la Ciencia y la Cultura (ONU), quien considera imprescindible dirigir los procesos de la formación de los docentes hacia la diversidad, aspecto notorio y destacado dentro de la globalización. Al respecto, Vélez (2011) explica que la educación inclusiva se enmarca en un modelo social, a partir del cual las barreras para el aprendizaje y la participación están en el entorno, siendo este el responsable de minimizarlas o abolirlas. Visto de esta manera, las dificultades están fundamentalmente en el currículo, no en el estudiante.

Las implicaciones educativas tienen que ver con la valoración de la diversidad como condición humana, y con la diferencia como un aspecto inherente a todos los seres humanos. Por ello, todos los sujetos se entienden como diversos. La responsabilidad del proceso educativo es de toda la comunidad educativa. La educación inclusiva supone, entonces, un cambio social en la manera de concebir las prácticas, las culturas y las políticas educativas y escolares (Vélez, 2001, p.7).

En este caso es necesario comprender que la diversidad implica la aceptación y el respeto por la diferencia del ser humano, lo cual es muy importante dentro de 
la educación, pues parte del principio de aceptar al otro independientemente de su condición en un contexto social. Esta afirmación conlleva un desafío para los futuros docentes de inglés, porque a través de su trabajo en el aula pueden generar situaciones en las cuales todos sus estudiantes sean tenidos en cuenta, respetando su diversidad.

Aunque es necesario reconocer y aceptar que en la actualidad la situación ha cambiado mucho, especialmente para los estudiantes, también es cierto que aún existe en la escuela un rechazo muy marcado por aquellos estudiantes que presentan alguna condición, no homogénea, para el resto del grupo dentro del cual hace su proceso de aprendizaje. Es claro que ayuda bastante considerar la inclusión dentro de los derechos humanos, razón por la cual es un tema de bastante interés no solamente dentro de la escuela, sino dentro de otros órganos de la sociedad.

Con este propósito, es necesario generar interés y motivación por parte del futuro docente de inglés, de tal manera que este asunto se convierta en un eje de investigación para ser impulsado dentro del proceso de enseñanza-aprendizaje de sus estudiantes.

Por esta razón, es indispensable que el futuro profesor de inglés proponga principios promotores de una sana convivencia, donde no haya lugar para la discriminación, sino por el contrario, que sus situaciones de enseñanza sean de inclusión y respeto por el otro.

En este sentido, Vélez (2001) señala que la educación debe dirigir la mirada hacia aspectos tales como el acceso, la eficiencia, la equidad, el género, la calidad y la relevancia, consideraciones poco destacadas durante los procesos de formación del docente de inglés, pese a ser de gran importancia.

En cierto modo, esto nos permite resaltar cómo, a través de la globalización, es posible que las personas tengan acceso a la información, a los medios y a las redes, rompiendo todo factor de exclusión y discriminación por la raza, el sexo o las creencias que, en otros tiempos, impedían a las personas el acceso a algunos medios de comunicación.

Adicionalmente, este autor menciona que la autonomía es otro elemento fundamental en la formación del docente de inglés en el contexto global, manifestada por el dominio y la capacidad del profesor para generar estrategias de formación, frente a los acontecimientos sucedidos en la escuela, buscando así, encontrar respuesta a las preguntas, como resultado del autocuestionamiento respecto a su proceso pedagógico.

Esta situación, probablemente, lo llevará a formarse como un nuevo ciudadano, con capacidad de enfrentarse a la globalización, con alto sentido ético y gran responsabilidad ante el significado de la autonomía, que le permitan ejercer su independencia de 
pensamiento, de comprensión y de actuación, así como proponer ideas y soluciones diferentes, de cara a las necesidades de la sociedad actual.

Es evidente que la autonomía debe estar acompañada de un proceso de reflexión crítica, con lo cual se espera que el futuro profesor de inglés desarrolle ideas a la luz de los hallazgos en el desarrollo de su práctica docente, especialmente.

Para alcanzar esta meta, el profesor debe contar con un gran bagaje teórico que le permita resolver las dudas que se le presenten en su práctica, pero que, a la vez, lo lleve a plantearse nuevas teorías, tomando en consideración las problemáticas halladas durante su inmersión en la escuela.

\subsection{Los retos en la formación del docente de inglés}

Los procesos de formación recibidos por el docente de inglés se convierten, como señala Fernández (2003), en retos. De acuerdo con este autor, "hay ciertas tendencias en la naturaleza de los saberes que la universidad gestiona y que se deben considerar, ya que constituyen verdaderos retos que la sociedad del conocimiento plantea a la enseñanza y el aprendizaje universitarios" (p. 275). Dichos retos se consideran, en primer lugar, desde el contexto global; es decir, para todos los agentes que intervienen en la escuela, cuyo propósito es producir un conocimiento útil cuando el docente de inglés en proceso de formación se acerque a la escuela. De igual manera, implica elaborar teorías frente a las posibles situaciones presentadas al docente en la escuela, de tal modo que su ejercicio sea el reflejo de una teoría, producto de su reflexión y crítica permanente.

En segundo lugar, desde una mirada económica, es necesario que el futuro docente de inglés esté formado para ser competente frente a los requerimientos del espacio dentro del cual ejercerá su labor pedagógica. En este sentido, debe estar muy atento a los perfiles requeridos por la escuela que funciona dentro de la globalización, para que pueda atender diversas situaciones.

En tercer lugar, desde la visión de sociedad, es necesario formar docentes de inglés críticos, reflexivos, propositivos y proactivos, con el propósito de brindar soluciones a las necesidades e inquietudes planteadas por la globalización, la cual ha producido una nueva generación con condiciones completamente diferentes a las vividas por los docentes. Por lo tanto, la sociedad, la escuela, sus estudiantes y los padres de familia tendrán otras expectativas de vida, las cuales deberán ser satisfechas por los docentes.

Finalmente, desde una visión ética, es importante que el futuro docente de inglés sea formado en la reconstrucción de valores, que sea fortalecedor del valor por lo propio 
(Ramos \& Aguirre, 2016), por lo autóctono, por las tradiciones y las costumbres, que facilite el desarrollo de una identidad y una idiosincrasia distintas a las de los demás seres del planeta.

Teniendo en cuenta lo expuesto, al profesor de inglés en proceso de formación se le debe brindar una adecuada preparación respecto al uso de las tecnologías, al análisis social y económico de la sociedad y al rescate de valores, que lo ponga en contacto con espacios y tiempos que le posibiliten poner en práctica los conocimientos adquiridos, y lo lleve a ser consciente de la relevancia del proceso en el cual se encuentra involucrado, y de la inmensa responsabilidad que tiene por demostrar un desempeño profesional que responda a las necesidades locales y globales del mundo actual.

Esta formación también debe considerar la interacción del maestro con su cultura, generada especialmente por su condición de profesor de lengua. Por esta razón, el profesor de inglés tiene la responsabilidad de aproximarse a la cultura correspondiente a la lengua enseñada, sin olvidar las tradiciones, creencias y costumbres propias, con el propósito de establecer comparaciones y generar un afianzamiento de los rasgos propios de la cultura correspondiente a su lengua nativa. Consecuentemente, el docente de inglés desempeñará un rol importante en las relaciones establecidas con sus estudiantes, para hacer que ellos sean conscientes de los valores culturales de la lengua materna frente a la cultura ofrecida por la lengua extranjera; proceso que debe estar plenamente acompañado de un ejercicio crítico, reflexivo, cambiante e interaccional.

De este modo, como lo establecen Goodson et al. (citados por Castro, Méndez \& Sercu, 2003), el docente toma distancia y entiende desde la perspectiva del "otro" su propia realidad cultural. Es innegable que el futuro docente de inglés establece una interacción con la comunidad académica dentro de la cual ejerce su labor, así como con su saber. Esto lo lleva a constituir un soporte pedagógico que probablemente lo conducirá a una mejor competencia como docente, haciéndolo más consciente de su desempeño, de las actividades realizadas con sus estudiantes, y de todos los demás aspectos desarrollados alrededor de los acontecimientos ocurridos, dentro o fuera del contexto escolar.

Esta condición también facilitará el fortalecimiento de habilidades, capacidades y desempeños de los futuros profesionales de cambio, quienes tendrán siempre presente la importancia de estar en permanente actualización de sus conocimientos, teniendo en cuenta las problemáticas sociales de la comunidad, de tal manera que pueda educar en el respeto y la integridad, sin olvidar la conservación del planeta para las generaciones futuras. 


\section{Discusión e implicaciones pedagógicas}

Teniendo en cuenta lo anterior, vale la pena resaltar que el futuro docente de inglés debe ser formado empleando los nuevos contextos de enseñanza y utilizando otros espacios que sean mucho más novedosos, amigables y efectivos en la construcción de una enseñanza mucho más significativa y pertinente para el aprendiz.

Además, se puede agregar que el estudiante formado para ser profesor de inglés debe estar preparado para enfrentar estos retos educativos, especialmente por lo novedoso de estos modernos contextos de enseñanza. En este sentido, debe estar dispuesto a recibir y dar el conocimiento en cualquiera de los escenarios que se le presenten.

Para el caso particular de la formación de los docentes de inglés, es innegable la existencia de múltiples herramientas que apoyan su labor y lo conducen a recrear sus espacios de enseñanza en contextos mucho más amplios y diversos. Por lo tanto, debe romper con la idea tradicional de que únicamente en el aula de clase se genera conocimiento, pues esta era ofrece nuevas condiciones, lugares y momentos para enseñar y aprender. Esto, obviamente, lo lleva a sobrepasar el ambiente ofrecido por el aula y a crear escenarios diferentes, con los cuales se eliminan las fronteras del contexto físico del salón de clase.

Las entidades formadoras de docentes no deben ser ajenas a estos cambios y, por el contrario, deben ofrecer una formación que conduzca a sus estudiantes a comprender que el escenario del aula de clase ya no está situado únicamente dentro de cuatro paredes, sino que va más allá, pues existen, por ejemplo, los entornos mediáticos, en los cuales también se pueden dar los procesos de formación de los docentes de inglés.

En consecuencia, es necesario que los futuros maestros tengan la capacidad de ser consecuentes con los cambios presentados y puedan reorientar los procesos metodológicos que acompañen su futuro ejercicio. Es decir, los profesores deben adaptarse y responder a los cambios en los procesos de enseñanza, así como asumir nuevos roles, con el fin de proponer alternativas frente a dichos cambios y gestar nuevas teorías facilitadoras de la organización y el funcionamiento de la sociedad, bajo las nuevas condiciones de la globalización.

Estos cambios llevan al docente de inglés en proceso de formación a plantear nuevos conocimientos, buscando siempre la forma de aplicarlos a la realidad del aula de clase. Debido a esto, las instituciones formadoras de docentes de inglés deben brindar una educación acorde a los requerimientos del nuevo contexto global, que está ansioso 
de profesores innovadores, creativos y propositivos, capaces de diseñar estrategias, materiales y metodologías diferentes, que sean interesantes para quienes acuden a adquirir el conocimiento.

En efecto, los procesos de formación ofrecidos al docente de inglés deben estar acompañados del conocimiento, la experiencia y la reflexión constante de quien se forma y de quien lo forma. De esta manera, se promueve el proceso de comprensión y reflexión de su actividad pedagógica, viabilizada por asumir una posición crítica que lo llevará a construir un conocimiento basado en su experiencia y en sus teorías, en contraste con las realidades de la práctica en la escuela.

En este sentido, se debe reconocer que el proceso de reflexión involucra tanto las ideas que se crean alrededor de un suceso pedagógico, como las acciones surgidas de este fenómeno del pensamiento, en la búsqueda de una transformación del ejercicio docente hecho en la escuela. Por lo tanto, se espera que el desempeño como profesor practicante de inglés tenga una incidencia en la sociedad.

Esto nos lleva a considerar que el profesor debe razonar acerca de los hechos sucedidos en la clase, estudiar y poner en marcha las ideas surgidas de sus reflexiones. De ese modo, conseguirá tener una incidencia altamente efectiva en el contexto escolar.

En consecuencia, se espera que la reflexión pedagógica tenga un amplio impacto en los fenómenos sociales, históricos y pedagógicos ocurridos dentro de la sociedad en la cual se encuentra el futuro profesor de inglés, de tal manera que lleve al salón de clase las situaciones cotidianas vividas tanto por los estudiantes, como por los profesores.

Esto se suscita, especialmente, cuando el futuro profesor de inglés se encuentra en su práctica docente, la cual propicia ejercicios de reflexión, crítica y transformación de su ejercicio pedagógico. En consecuencia, se espera que la reflexión crítica conduzca al futuro docente de inglés a desarrollar actividades consideradas como invaluables oportunidades de cuestionamiento acerca de sus creencias y concepciones sobre su papel en la sociedad. Esto quiere decir que, en la actualidad, se espera que el profesor asuma roles distintos a los tradicionales.

En fin, vale la pena terminar afirmando que la reflexión ha jugado un papel importante en el campo de la pedagogía y, actualmente, desempeña un papel de mucho más valor dentro del fenómeno de la globalización. En esta era, la reflexión y la crítica han ido tomando mayor fuerza en los nuevos procesos de formación del profesor de inglés, e igualmente el compromiso de los docentes y los estudiantes se ha incorporado de forma 
más amplia para trabajar como eslabones en el gran engranaje de aprender y enseñar mutuamente.

\section{Conclusiones}

Todas estas consideraciones nos conducen a afirmar que el nuevo docente, formado en el marco de la globalización, debe integrar el conocimiento que tiene y que está adquiriendo dentro de su proceso de formación, en la práctica. En tal sentido, en la práctica, el futuro docente de inglés tendrá la oportunidad de analizar herramientas, técnicas, lineamientos pedagógicos, preceptos políticos, teorías y principios aprendidos hasta ese momento, para demostrar el dominio conceptual de los contenidos correspondientes al saber enseñado. Adicionalmente, tendrá que ser un líder con un gran manejo del grupo, que genere confianza y promueva el trabajo en comunidad con sus estudiantes, lleno de valores, especialmente de respeto y capacidad de aceptación de la diferencia en un mundo tan dispar como el nuestro. Así, el profesor de inglés mostrará la capacidad de tomar decisiones acertadas frente a cualquier situación que se presente dentro o fuera del aula.

En resumen, el futuro profesor de inglés debe demostrar tener un conocimiento de la lengua y de todos los aspectos relacionados con el manejo didáctico en el salón de clase y fuera de él, manejar una comunicación adecuada con sus estudiantes, ser creativo y estar en capacidad de reflexionar sobre su actividad pedagógica, con miras a convertirse en un aprendiz continuo del proceso ejecutado, para poder ser un investigador de su actividad pedagógica y lograr grandes cambios en la realidad donde interviene como docente e investigador.

Es muy importante que el futuro docente de inglés maneje sus emociones, sus afectos y sus sentimientos, para que pueda tener la capacidad de tomar decisiones sin ser subjetivo, y dar opiniones de sus estudiantes desde una perspectiva realista, concreta y, ante todo, académica.

Este esfuerzo deberá convertirlo en un profesor autónomo, líder de su propio proceso de formación, pendiente de todas las situaciones o hechos ocurridos en la realidad donde hace su trabajo pedagógico, con la seguridad de tomar decisiones basadas en las indagaciones de sus investigaciones, de tal manera que su actitud lo lleve a grandes cambios en la escuela.

Por tal razón, el futuro docente de inglés debe poseer una amplia formación ciudadana que promueva los valores en sus estudiantes y lo lleve a tener un mejor 
propósito de vida, así como un desempeño acorde con la realidad de las instituciones escolares.

A manera de conclusión, la globalización exige la búsqueda de nuevas alternativas para la formación de profesores de inglés en el contexto global. Dentro de estas existen elementos claves como estar en una comunidad académica, ser reflexivo, teórico, creativo e innovador en sus procesos de enseñanza y aprendizaje, los cuales nunca terminarán, mientras siga siendo un docente.

En definitiva, el futuro docente de inglés está llamado a pasar de la teoría a la práctica a través de la producción de sus propias metodologías y postulados. De este modo, la práctica ya no será un proceso de imitación 0 implementación de técnicas 0 estrategias, sino que se convertirá en un proceso de constante observación, evaluación y adaptación, basado en los componentes teóricos aprendidos durante su proceso de formación. Lo anterior le permitirá articular la teoría con la práctica y producir nuevos constructos teóricos, mediante la reflexión y el cuestionamiento permanente.

\section{Referencias}

Biggs, J. (2006). Calidad de aprendizaje universitario. Madrid: Narcea.

Brunner, J. (2001). Perspectivas desde el siglo XXI. Perspectivas, 4(2), 203-211.

Cabero, J. (2004). Nuevas tecnologías en la práctica educativa. Granada: Arial.

Castro, P., Méndez, M. \& Sercu, L. (2003). La cultura en el aula de lenguas extranjeras: análisis de las percepciones de los profesores de secundaria respecto a los objetivos y las actividades de aprendizaje cultural. Recuperado de: http://sedll.org/es/admin/uploads/ congresos/15/act/131/Castro_Prieto.pdf

Fernández, l. A. (2003). El docente universitario. Retos y perspectivas en los inicios del siglo XXI. En Congreso Internacional de Investigación Educativa ILMEC-INIE 25 años en Pro de la Educación. Recuperado de: http://www.inie.ucr.ac.cr/congreso/memoria/archivos/ ponencias/jorgefernandez2.pdf

García León, D. \& García León, J. (2014). Educación bilingüe y pluralidad: reflexiones en torno de la interculturalidad crítica. Cuadernos de Lingüística Hispánica, (23), 49-65.

Hallak, J. (2008). Globalización, derechos humanos y educación. París, Estocolmo: Instituto Internacional de Planeamiento de la Educación, UNESCO.

Jiménez Niño, P. (2014). Exploring students' reactions when working teaching materials designed on their own interests. Cuadernos de Lingüística Hispánica, (25), 201-222. 
Kumaravadivelu, B. (2003). Beyond Methods: Macrostrategies for Language Teaching. New Haven: Yale University press.

Kumaravadivelu, B. (2006). Understanding Language Teaching. From Method to Postmethod. Mahwah, NJ: Lawrence Erlbaum Associates.

León, G. (2004). La educación en el contexto de la globalización. Revista Historia de la Educación Latinoamericana, 6.

Peña, J. (2003, oct.-nov.). Desarrollo profesional del docente universitario. Monografías Virtuales, Ciudadania, Democracia y Valores en Sociedades Plurales, (3). Recuperado de: http:// www.oei.es/valores2/monografias/monografia03/reflexion03.htm.

Ramos, B. \& Aguirre, J. (2016). English Language Teaching in Rural Areas: A New Challenge for English Language Teachers in Colombia. Cuadernos de Lingüística Hispánica, (27), 209- 222.

Sánchez, J. (s. f.). El desarrollo profesional del docente universitario. Recuperado de: http:// www.udual.org/CIDU/Revista/22/DesarrolloProfesional.htm.

Vélez, L. (2011). La educación inclusiva en los programas de formación inicial de docentes. (Proyecto de investigación doctoral). Doctorado Interinstitucional en Educación. Universidad Pedagógica Nacional, Bogotá.

Zabalza, M. A. (2004). A Didáctica Universitaria. Un espazo disciplinar para o estudo e mellora da nosa docencia. Santiago de Compostela: Universidad de Santiago de Compostela. 